\title{
Urine phyto-oestrogen metabolites are not significantly associated with risk of type 2 diabetes: the Singapore Chinese health study
}

\author{
Mohammad Talaei ${ }^{1}$, Bee L. Lee ${ }^{1}$, Choon N. Ong ${ }^{1}$, Rob M. van Dam ${ }^{1,2,3}$, Jian M. Yuan ${ }^{4,5}$, Woon P. Koh ${ }^{6,1}$ \\ and An Pan ${ }^{7 *}$ \\ ${ }^{1}$ Saw Swee Hock School of Public Health, National University of Singapore and National University Health System, Singapore \\ 117549, Singapore \\ ${ }^{2}$ Yong Loo Lin School of Medicine, National University of Singapore and National University Health System, Singapore 119228, \\ Singapore \\ ${ }^{3}$ Department of Nutrition, Harvard T.H. Chan School of Public Health, Boston, MA 02115, USA \\ ${ }^{4}$ Division of Cancer Control and Population Sciences, University of Pittsburgh Cancer Institute, Pittsburgh, PA 15232, USA \\ ${ }^{5}$ Department of Epidemiology, University of Pittsburgh Graduate School of Public Health, Pittsburgh, PA 15232, USA \\ ${ }^{6}$ Duke-NUS Graduate Medical School, Singapore 169857, Singapore \\ ${ }^{7}$ Department of Epidemiology and Biostatistics, MOE Key Laboratory of Environment and Health, School of Public Health, \\ Tongji Medical College, Huazhong University of Science and Technology, Wuhan, Hubei 430030, People's Republic of China
}

(Submitted 3 April 2015 - Final revision received 25 November 2015 - Accepted 19 January 2016 - First published online 7 March 2016)

\section{Abstract}

We evaluated the relationship between urine concentrations of phyto-oestrogens (isoflavones and lignans) and risk of incident type 2 diabetes in middle-aged and elderly Chinese residing in Singapore. Urine metabolites of isoflavones and lignans were assayed by HPLC among 564 diabetes cases and 564 matched controls in a case-control study nested within the Singapore Chinese Health Study cohort. Participants were free of diagnosed diabetes, CVD and cancer at morning urine collections during 1999-2004. Cases were participants who reported to have physician-diagnosed diabetes at follow-up visits during 2006-2010, whereas controls were randomly selected among those who remained free of diabetes and were matched to the index cases by age, sex, dialect group and date of urine collection. Conditional logistic regression models were used to calculate OR and $95 \%$ CI with adjustment for potential confounders. The mean age of the participants at the time of urine collection was $59 \cdot 8$ years, and the average interval between urine collection and diabetes diagnosis was 4.0 years. The multivariate-adjusted OR for diabetes were 1.00 (reference), 0.76 (95\% CI $0.52,1 \cdot 11), 0.78$ (95\% CI 0.53, 1.14) and 0.79 (95\% CI 0.54, 1.15) across quartiles of urine isoflavones $\left(P_{\text {for trend }}=0.54\right)$, and were 1.00 (reference), 0.87 (95\% CI 0.60, 1.27), 1.10 (95\% CI $\left.0.77,1.56\right)$ and 0.93 $(95 \%$ CI $0.63,1.37)$ for lignans $\left(P_{\text {for trend }}=0.93\right)$. The results were similar in men and women, as well as for individual metabolites of isoflavones (genistein, daidzein, glycitin and equol) or lignans (enterodiol and enterolactone). The present study did not find a significant association between urine phyto-oestrogen metabolites and risk of type 2 diabetes in Chinese adults.

Key words: Type 2 diabetes: Isoflavones: Lignans: Urine metabolites: Incidence

The historically low prevalence of type 2 diabetes (T2D) in populations with high consumption of soya, legumes and whole grains has raised interest in the potential protective role of these foods in the development of $\mathrm{T} 2 \mathrm{D}^{(1,2)}$. Phyto-oestrogens, with isoflavones and lignans as the major types, are among the potentially active components in these food groups ${ }^{(3)}$. Isoflavones are concentrated in soyabeans and soya products ${ }^{(3)}$, whereas lignans are mainly concentrated in flaxseed, sesame seeds, whole-grain and other plant-based foods ${ }^{(4)}$. It has been hypothesised that these compounds could impose anti-diabetic effect through several mechanisms including pathways that are dependent or independent of their oestrogenic properties ${ }^{(3-6)}$.
Despite promising results from experimental studies on the potentially beneficial effects of isoflavones and lignans on glucose metabolism ${ }^{(3-6)}$, findings from human observational studies are contradictory regarding the association between their dietary intakes and risk of $\mathrm{T} 2 \mathrm{D}^{(7-11)}$. The inconsistent findings might be due to the difficulty in accurately measuring dietary intakes of isoflavones and lignans in epidemiological studies. The lack of comprehensive, accurate and up-to-date food composition databases is the major shortcoming due to the large variability of these compounds in diverse food items, which are influenced by farming conditions, post-harvest processing, storage conditions and cooking methods ${ }^{(12)}$.

Abbreviations: CRP, C-reactive protein; NHS, Nurse's Health Study; SCHS, Singapore Chinese Health Study; T2D, type 2 diabetes.

* Corresponding author: A. Pan, email panan@hust.edu.cn 
Moreover, dietary isoflavones and lignans need to undergo substantial metabolism by intestinal microbiota to produce the bioactive forms ${ }^{(3,4)}$. The inter-individual variations in the metabolism of these compounds add further complexity to accurately quantify the biological effects of phyto-oestrogens on human health.

A recent study in US women found that higher urine concentrations of lignan metabolites were associated with a $30 \%$ lower risk of T2D for each standard deviation increase ${ }^{(13)}$; however, this relation has not been evaluated in Asian populations where high consumption of isoflavones from soya and soya products are common. To the best of our knowledge, evidences about the relationship between urine metabolites of phyto-oestrogens and risk of T2D are scarce. Therefore, in the present study, we aimed to measure urine metabolites of isoflavones and lignans as biomarkers of dietary intake of phyto-oestrogens and to examine their relations with risk of T2D among Chinese adults residing in Singapore.

\section{Methods}

\section{Study population}

The design of the Singapore Chinese Health Study (SCHS) has been described in detail previously ${ }^{(14)}$. In brief, the SCHS is a prospective cohort study that was established between 1993 and 1998. The study recruited 63257 Chinese men and women, between 45 and 74 years of age, from two major dialect groups (Cantonese and Hokkiens) of Chinese in Singapore. All participants were citizens and permanent residents who lived in government-built housing estates ( $86 \%$ of Singapore residents lived in such estates at that time). The present study has been approved by the Institutional Review Boards at the National University of Singapore, and informed consent was provided with completion of the baseline interview.

At recruitment between 1993 and 1998, trained interviewers administered structured questionnaires for each study participant through face-to-face interviews at home, and collected information on usual diet, demographics, height and weight, smoking and drinking habits, usual physical activity, menopausal status and medical history including physician-diagnosed diabetes, hypertension, CHD and stroke. History of diagnosed cancer was assessed via linkage with the nationwide Singapore Cancer Registry. A validated 165 -item, semi-quantitative FFQ was used to assess habitual dietary habits over the previous year ${ }^{(14)}$, and the Singapore Food Composition Table was developed based on raw and cooked foods consumed in this population to compute intakes of nutritive/non-nutritive dietary components. The food groups were adjusted for energy intake using the energy-density method, and food intakes were expressed as $\mathrm{g} /(1000 \mathrm{kcal} \times \mathrm{d})$ $(1 \mathrm{kcal}=4 \cdot 2 \mathrm{~kJ})$.

Between 1999 and 2004, a total of 52325 participants were re-contacted via telephone to update certain lifestyle practices (e.g. smoking habits and alcohol consumption), body weight and medical history (e.g. diabetes, hypertension, CHD and stroke) during the follow-up I interview. They were also invited to donate their biospecimen samples, and 32535 participants agreed and gave their morning blood and spot urine samples.
Details of the biospecimen collection, processing and storage procedures are explained elsewhere ${ }^{(15)}$. Spot urine samples were obtained at subjects' home in the morning. The urine was first collected in 250-ml plastic jars and immediately kept on ice. Within $1 \mathrm{~h}, 100 \mathrm{ml}$ urine was acidified with $2 \mathrm{~g}$ of ascorbic acid in a conical flask. Subsequently, it was aliquoted into five vials $(20 \mathrm{ml})$ and stored at $-70^{\circ} \mathrm{C}$ until analysis ${ }^{(16)}$. Participants ( $n$ 39528) were re-contacted again in 2006-2010 (follow-up II interview) to update selected lifestyle habits and health status.

\section{Assessment of diabetes}

In all the interviews at recruitment, follow-up I and II, participants were asked 'Have you been told by a doctor that you have diabetes (high blood sugar)?', and in case of a positive answer, they were additionally enquired the age of first diagnosis. A validation study of the incident diabetes cases was reported in detail previously ${ }^{(17)}$. On the basis of a hospitalbased discharge summary database and a supplementary questionnaire regarding symptoms, diagnostic tests and hyperglycaemic therapy during a telephone interview, we observed a positive predictive value of $99 \%{ }^{(17)}$. Thus, the self-reported history of diabetes is a highly reliable measure of diagnosed diabetes status in this population.

\section{Case and control selection}

The present study samples were chosen from a case-control genome-wide association study of T2D nested within the $\mathrm{SCHS}^{(18)}$. All participants without a diagnosis of T2D at baseline and follow-up I (before biospecimen collection), and who subsequently provided urine samples for research were eligible for the present study. We excluded participants with reported $\mathrm{CHD}$, stroke or those who had cancer at the time of biospecimen collection. We further excluded those who reported CHD or stroke or had cancer diagnosed before the diagnosis of diabetes for the selection of cases, and at follow-up II visits for the selection of controls. Cases were those who reported to have physician-diagnosed diabetes during the follow-up II visit - that is, they were diagnosed between follow-up I and follow-up II interviews. Controls were free of physiciandiagnosed T2D at recruitment and all follow-up interviews, and were matched for age ( \pm 3 years) and date ( \pm 6 months) of biospecimen collection, sex and dialect group to their index cases. In addition, controls were also screened for the presence of undiagnosed $\mathrm{T} 2 \mathrm{D}$ by ensuring a HbA1c level $<6.0 \%$ ( $42 \mathrm{mmol} / \mathrm{mol}$ ) at the time of biospecimen collection (online Supplementary Fig. S1). Finally, a total of 564 diabetes cases and 564 matched controls were chosen for this investigation.

\section{Laboratory measurements}

Case-control pairs of urine samples were analysed in the same batch. One senior technician with one assistant handled all assays under identical conditions without knowing the case-control sequences. Concentrations of six urine metabolites including isoflavones (daidzein, genistein, glycitein, and equol) and lignans (enterodiol and enterolactone) were measured through HPLC (Thermo Scientific Dionex UltiMate 3000 LC system) modified 
from an established LC-MS/MS method ${ }^{(19)}$ (online Supplementary Material). The within-day and between-day CV were $<15 \%$ for all metabolites except for the between-day CV of genistein (16.8\%). Urine creatinine was measured according to the Jaffe's method (reaction with alkaline picrate using an auto-analyser). Urine concentrations of phyto-oestrogen metabolites were expressed as $\mathrm{nmol} / \mathrm{mg}$ creatinine.

Frozen plasma aliquots were used to simultaneously quantify high-sensitivity C-reactive protein (CRP) and lipids (total cholesterol, TAG and HDL-cholesterol) using the colorimetric method on a chemistry analyzer (AU5800 Analyzer; Beckman Coulter) at the National University Hospital Reference Laboratory. Erythrocyte HbA1c was measured by HPLC method using Bio-Rad Variant II ${ }^{\mathrm{TM}}$ System (Bio-Rad Laboratories). The blood samples of index cases and matched controls were assayed in the same laboratory batch.

\section{Statistical analysis}

Characteristics were compared between T2D cases and their matched controls using paired Student's $t$ test for continuous variables and the $\chi^{2}$ test for categorical variables. Owing to skewness, natural logarithm transformation was performed for TAG, CRP, total energy intake, vegetable intake, fruits and related juices intake, soya protein and isoflavone intake, and urine isoflavones, lignans and creatinine concentrations. Backtransformed values are shown in the form of geometric mean and $95 \%$ CI. Conditional logistic regression was used to assess the relationship of quartiles of creatinine-adjusted urine phytooestrogens (total, isoflavones and lignans), isoflavones subclasses (daidzein, genistein, glycitein and equol) and lignans components (enterodiol and enterolactone) with the risk of incident T2D. Quartiles were defined based on the distribution of urine concentrations of isoflavones and lignans in the control group. Statistical significance of linear trends was tested by modelling the median of each quartile as a continuous variable.

In the multivariate analysis, we first adjusted for age at biospecimen collection (continuous) and fasting status ( $\geq 6$ or $<6 \mathrm{~h}$ after meal); we then further adjusted for education level (no, primary school and less, secondary school and above), BMI $\left(<20 \cdot 0,20 \cdot 0-23 \cdot 9,24 \cdot 0-27 \cdot 9\right.$ and $\left.\geq 28 \cdot 0 \mathrm{~kg} / \mathrm{m}^{2}\right)$, physical activity $(<0.5,0.5-3.9$ and $\geq 4.0 \mathrm{~h} /$ week), smoking (never, former and current), alcohol use (no/occasionally, weekly and daily), history of hypertension (yes/no) and total energy intake (continuous). In the sex-specific analysis, we also adjusted for menopausal status (yes/no) in women. In the final model, we additionally adjusted for other dietary variables including quartiles of total vegetables, total fruits and related juices, SFA and $n-3$ fatty acids. The information on education level, physical activity and dietary intake was obtained from baseline interviews (1993-1998), and the other variables were from follow-up I visits (1999-2004), which were close to the urine sample collection. In a sensitivity analysis, we further adjusted for sweetened soya items as well as plasma CRP, TAG and HDL-cholesterol. We also used restricted cubic spline regression with 4 knots to detect potential non-linear association, and we used the likelihood ratio test to assess non-linearity by comparing models with and without the cubic spline term.
An HbA1c level higher than $6.5 \%(47.5 \mathrm{mmol} / \mathrm{mol})$ was first proposed as diabetes diagnostic criteria in 2009 by the International Expert Committee ${ }^{(20)}$ and was later adapted by the American Diabetes Association (ADA) $)^{(21)}$ as a diagnostic criterion. Therefore, HbA1c was not used as a diagnostic criterion of diabetes at the time of biospecimen collection (2000-2004). However, some of the incident T2D cases already had $\mathrm{HbA} 1 \mathrm{c} \geq 6.5 \%(47.5 \mathrm{mmol} / \mathrm{mol})$ at the time of biospecimen collection, and could thus be classified as undiagnosed diabetes based on the current ADA diagnostic criteria. To account for this definition development, a sensitivity analysis was carried out by stratifying case-control pairs based on HbA1c $<6.5 \%$ or $\geq 6.5 \%$ in the case groups at the time of biospecimen collection. Statistical analyses were carried out by Stata Release 11.2 (StataCorp LP). For all analyses, statistical significance was assessed at a level of 0.05 (two-tailed).

\section{Results}

The mean time between urine collection and diabetes diagnosis was 4.0 years (range $1-9$ years). Table 1 shows the characteristics of cases and controls at biospecimen collection (while some variables were from baseline interviews). The mean age of the participants was 59.8 years at biospecimen collection. As expected, cases had a high-risk profile except for the matching factors. They were heavier and more likely to have hypertension, had higher blood levels of TAG and CRP, but lower HDL-cholesterol. In terms of soya food intake, diabetes cases consumed significantly lower soya protein and isoflavones compared with the controls. However, no significant differences were found with respect to urine isoflavones or lignans. In the controls, the mean concentration of urine isoflavones was $4.01 \mathrm{nmol} / \mathrm{mg}$ creatinine, and daidzein and genistein were the major forms; the mean concentration of urine lignans was $1.39 \mathrm{nmol} / \mathrm{mg}$ creatinine, and enterolactone was the major form.

Among the control subjects, no significant associations were found between the covariates and total urine isoflavones (data not shown). However, those with higher total urine lignan concentrations were more likely to be female, had higher physical activity and SFA intake, but a lower prevalence of hypertension and daily alcohol consumption (data not shown).

We also tested the association between dietary soya intake at the baseline interview from 1993 to 1998 and urine isoflavone metabolites in urine samples collected from 1999 to 2004. The average time between the baseline dietary assessment and biospecimen collection was 6.5 (SD 1.6) years. A dose-dependent positive relation was observed between soya protein intake and total urine isoflavones. The same trend was observed for soya isoflavone intake (Table 2).

Table 3 presents the relationships of total urine phytooestrogens, isoflavones and lignans with T2D risk. No statistically significant association or trend was observed for any of the exposures. The results were not materially changed after further adjustment for sweetened soya items, blood lipids (HDL-cholesterol and TAG) and CRP (data not shown). Introducing creatinine variable to the statistical models as a confounder rather than adjusting creatinine concentration for individual phyto-oestrogen metabolites did not materially change the results 
Table 1. Characteristics of the study participants in diabetes cases and control group

(Numbers and percentages; mean values and standard deviations; geometric means and $95 \%$ confidence intervals)

\begin{tabular}{|c|c|c|c|c|c|}
\hline & \multicolumn{2}{|c|}{ Controls ( $n$ 564) } & \multicolumn{2}{|c|}{ T2D cases $(n 564)$} & \multirow[b]{2}{*}{$P^{*}$} \\
\hline & $n$ & $\%$ & $n$ & $\%$ & \\
\hline Sex (female) & 329 & $58 \cdot 3$ & 329 & $58 \cdot 3$ & - \\
\hline Menopause & 192 & $58 \cdot 4$ & 199 & $60 \cdot 5$ & 0.57 \\
\hline Education level & & & & & 0.28 \\
\hline No formal school & 98 & 17.4 & 103 & $18 \cdot 3$ & \\
\hline Primary school & 230 & 40.8 & 251 & 44.5 & \\
\hline Secondary school & 236 & 41.8 & 210 & $37 \cdot 2$ & \\
\hline Hypertension & 148 & $26 \cdot 4$ & 262 & $46 \cdot 4$ & $<0.001$ \\
\hline Cigarette smoking & & & & & 0.18 \\
\hline Never & 419 & $74 \cdot 3$ & 404 & $68 \cdot 3$ & \\
\hline Former smokers & 70 & $10 \cdot 0$ & 63 & $10 \cdot 7$ & \\
\hline Current smokers & 75 & $30 \cdot 3$ & 97 & $21 \cdot 0$ & \\
\hline Alcohol intake (g/d) & & & & & 0.81 \\
\hline None/occasionally & 490 & $86 \cdot 9$ & 491 & $87 \cdot 1$ & \\
\hline Weekly & 59 & 10.5 & 55 & $9 \cdot 7$ & \\
\hline Daily & 15 & $2 \cdot 7$ & 18 & 3.2 & \\
\hline Physical activity (strenuous sports) & & & & & 0.12 \\
\hline None & 447 & $79 \cdot 3$ & 450 & $79 \cdot 8$ & \\
\hline$<4 \mathrm{~h} /$ week & 68 & $12 \cdot 1$ & 81 & 14.4 & \\
\hline$\geq 4 \mathrm{~h} /$ week & 49 & 8.7 & 33 & $5 \cdot 6$ & \\
\hline \multirow[t]{2}{*}{ Fasting duration $>6 \mathrm{~h}$} & 152 & $26 \cdot 9$ & 176 & $31 \cdot 2$ & 0.12 \\
\hline & Mean & SD & Mean & SD & \\
\hline Age at biospecimen collection (years) & 59.8 & $6 \cdot 2$ & $59 \cdot 7$ & $6 \cdot 1$ & 0.21 \\
\hline BMI $\left(\mathrm{kg} / \mathrm{m}^{2}\right)$ & $22 \cdot 7$ & 2.9 & 24.5 & 3.4 & $<0.001$ \\
\hline SFA $(g /(1000 \mathrm{kcal} \times d)) \dagger \ddagger$ & 8.97 & $2 \cdot 6$ & 9.13 & $2 \cdot 6$ & 0.34 \\
\hline$n-3$ Fatty acid $(g /(1000 \mathrm{kcal} \times \mathrm{d})) \dagger \ddagger$ & 0.54 & 0.18 & 0.52 & 0.18 & 0.04 \\
\hline \multicolumn{6}{|l|}{ Plasma biomarkers } \\
\hline Total cholesterol (mmol/l) & $5 \cdot 20$ & 0.8 & $5 \cdot 31$ & 0.9 & 0.052 \\
\hline \multirow[t]{2}{*}{ HDL-cholesterol (mmol/l) } & 1.23 & 0.3 & 1.08 & 0.2 & $<0.001$ \\
\hline & Mean & $95 \% \mathrm{Cl}$ & Mean & $95 \% \mathrm{Cl}$ & \\
\hline \multicolumn{6}{|l|}{ Plasma biomarkers } \\
\hline TAG (mmol/l) & 1.58 & $1.51,1.64$ & $2 \cdot 12$ & $2 \cdot 03,2 \cdot 21$ & $<0.001$ \\
\hline C-reactive protein (mg/dl) & $1 \cdot 17$ & $1.07,1.27$ & 1.90 & $1 \cdot 75,2 \cdot 06$ & $<0.001$ \\
\hline Total energy intake $(\mathrm{kcal} / \mathrm{d}) \dagger \ddagger$ & 1512 & 1470,1554 & 1555 & 1514,1598 & 0.11 \\
\hline All vegetables $(\mathrm{g} /(1000 \mathrm{kcal} \times \mathrm{d})) \dagger \ddagger$ & $69 \cdot 4$ & $66 \cdot 8,72 \cdot 1$ & $69 \cdot 2$ & $66 \cdot 7,71 \cdot 9$ & 0.84 \\
\hline All fruits and related juices $(g /(1000 \mathrm{kcal} \times d)) \dagger \ddagger$ & 113 & 105,122 & 105 & $97 \cdot 0,113$ & 0.15 \\
\hline Soya isoflavone intake $(\mathrm{mg} /(1000 \mathrm{kcal} \times \mathrm{d})) \dagger \ddagger$ & 9.86 & $9 \cdot 15,10 \cdot 6$ & 8.81 & $8.23,9.43$ & 0.03 \\
\hline Soya protein intake $(g /(1000 \mathrm{kcal} \times \mathrm{d})) \dagger \ddagger$ & 1.28 & $1 \cdot 19,1 \cdot 37$ & 1.18 & $1 \cdot 11,1 \cdot 26$ & $0 \cdot 10$ \\
\hline Total urine phyto-oestrogens ( $\mathrm{nmol} / \mathrm{mg}$ creatinine) & 7.35 & $6.72,8.03$ & 6.62 & $6.03,7.25$ & 0.11 \\
\hline Total urine isoflavones ( $\mathrm{nmol} / \mathrm{mg}$ creatinine) & 4.01 & $3.60,4.42$ & 3.71 & $3 \cdot 33,4 \cdot 15$ & 0.33 \\
\hline Daidzein (nmol/mg creatinine) & 1.43 & $1.26,1.61$ & 1.35 & $1.18,1.53$ & 0.52 \\
\hline Genistein (nmol/mg creatinine) & $1 \cdot 17$ & $1.06,1.29$ & 1.07 & $0.96,1.19$ & 0.25 \\
\hline Glycitein (nmol/mg creatinine) & 0.24 & $0.22,0.26$ & 0.23 & $0.21,0.25$ & 0.49 \\
\hline Equol (nmol/mg creatinine) & 0.08 & $0.06,0.10$ & 0.07 & $0.05,0.08$ & 0.36 \\
\hline Total urine lignans ( $\mathrm{nmol} / \mathrm{mg}$ creatinine) & 1.39 & $1.22,1.59$ & 1.23 & $1.08,1.40$ & 0.17 \\
\hline Enterodiol (nmol/mg creatinine) & 0.19 & $0.16,0.21$ & 0.17 & $0.15,0.19$ & 0.36 \\
\hline Enterolactone (nmol/mg creatinine) & 0.95 & $0.82,1 \cdot 10$ & 0.83 & $0.71,0.96$ & 0.20 \\
\hline Urine creatinine $(\mathrm{mg} / \mathrm{dl})$ & 66.7 & $63 \cdot 2,70 \cdot 3$ & $71 \cdot 8$ & $68 \cdot 0,75 \cdot 9$ & 0.04 \\
\hline
\end{tabular}

The cases and controls were matched for age ( \pm 3 years) and date ( \pm 6 months) of blood collection, sex and dialect group.

* $P$ values for case-control differences were calculated using paired Student's $t$ test for continuous variables and the $x^{2}$ test for categorical variables.

† A total of twelve pairs did not have data for the following dietary variables: total energy intake, all vegetables, all fruits and related juices, SFA, $n$ - 3 fatty acid, soya isoflavone and soya protein.

$\ddagger 1 \mathrm{kcal}=4 \cdot 2 \mathrm{~kJ}$.

(data not shown). Neither urine isoflavones nor lignans showed a statistically significant association with T2D risk when separately examined in men or women (online Supplementary Table S1). When we stratified the analysis by baseline HbA1c levels $(<6.5$ or $\geq 6.5 \%)$ in the diabetes cases, no significant trend was observed in either stratum (Table 4).
Table 3 suggests that the association between urine isoflavones and T2D risk was similar across the second to fourth quartiles; thus, we conducted a sensitivity analysis of non-linear association. Although no significant non-linearity was found $(P=0.37)$, the T2D risk was reduced at low concentrations and reached plateau afterwards (online Supplementary Fig. S2). This 
Table 2. Urinary isoflavones according to dietary intakes* of soya in all subjects

(Medians and interquartile ranges (IQR))

\begin{tabular}{|c|c|c|c|c|c|c|c|c|c|}
\hline & \multicolumn{8}{|c|}{ Quartiles of dietary intake $(\mathrm{Q})$} & \multirow[b]{3}{*}{$P_{\text {for trend }}{ }^{*}$} \\
\hline & \multicolumn{2}{|c|}{ Q1 } & \multicolumn{2}{|c|}{ Q2 } & \multicolumn{2}{|c|}{ Q3 } & \multicolumn{2}{|c|}{ Q4 } & \\
\hline & Median & IQR & Median & IQR & Median & IQR & Median & IQR & \\
\hline Soya protein intake $(\mathrm{g} /(1000 \mathrm{kcal} \times \mathrm{d})) \dagger$ & 0.59 & $0.39-0.73$ & $1 \cdot 15$ & $1.04-1 \cdot 30$ & 1.75 & $1.61-1.90$ & $2 \cdot 78$ & $2.43-3.32$ & \\
\hline Urinary isoflavones (nmol/mg creatinine) $\ddagger$ & 3.39 & $3 \cdot 11-3 \cdot 64$ & 3.89 & $3.57-4.23$ & 4.07 & $3.79-4.40$ & 4.41 & $4 \cdot 08-4.75$ & 0.03 \\
\hline Soya isoflavones intake $(g /(1000 \mathrm{kcal} \times d)) \dagger$ & 4.22 & $2 \cdot 69-5 \cdot 29$ & 8.77 & $7.49-9.90$ & 13.8 & $12 \cdot 4-15 \cdot 3$ & 22.0 & $19 \cdot 3-28 \cdot 2$ & \\
\hline Urinary isoflavones (nmol/mg creatinine) $\ddagger$ & $3 \cdot 47$ & $3 \cdot 17-3 \cdot 74$ & 3.81 & $3 \cdot 48-4 \cdot 11$ & $4 \cdot 21$ & $3.89-4.58$ & $4 \cdot 28$ & $3.94-4.57$ & 0.09 \\
\hline
\end{tabular}

* Linear trend was tested by treating the median of quartiles of intake as a continuous variable in a linear regression model adjusted for education level, BMI, physical activity, smoking status, alcohol use, hypertension history, total energy intake, vegetable intake, fruits and related juices intake, SFA and $n$-3 fatty acid to estimate log-transformed values of total isoflavone.

† Dietary intake data were derived from FFQ at baseline interviews (1993-1998), whereas urine samples were collected during follow-up I visits (1999-2004).

$\ddagger$ Values are adjusted for variables described in *.

Table 3. Incident type 2 diabetes according to urine phyto-oestrogen metabolites

(Odds ratios and $95 \%$ confidence intervals; medians and interquartile ranges (IQR))

\begin{tabular}{|c|c|c|c|c|c|c|c|c|}
\hline & \multicolumn{7}{|c|}{ Quartiles (Q) } & \multirow[b]{3}{*}{$P_{\text {for trend }}{ }^{*}$} \\
\hline & \multirow[b]{2}{*}{ Q1 } & \multicolumn{2}{|c|}{ Q2 } & \multicolumn{2}{|c|}{ Q3 } & \multicolumn{2}{|c|}{ Q4 } & \\
\hline & & OR & $95 \% \mathrm{Cl}$ & OR & $95 \% \mathrm{Cl}$ & OR & $95 \% \mathrm{Cl}$ & \\
\hline \multicolumn{9}{|l|}{ Total phytoestrogens } \\
\hline Median† & 1.99 & \multicolumn{2}{|c|}{5.04} & \multicolumn{2}{|c|}{$10 \cdot 3$} & \multicolumn{2}{|c|}{$25 \cdot 9$} & \\
\hline IQR & $1.29-2.62$ & \multirow{2}{*}{\multicolumn{2}{|c|}{$\begin{array}{c}4 \cdot 30-6 \cdot 15 \\
135 / 141\end{array}$}} & \multirow{2}{*}{\multicolumn{2}{|c|}{$\begin{array}{c}8 \cdot 73-12 \cdot 9 \\
149 / 141\end{array}$}} & \multicolumn{2}{|c|}{$19 \cdot 8-39 \cdot 1$} & \\
\hline Cases/control & $159 / 141$ & & & & & & $/ 141$ & \\
\hline Multivariate model $1 \ddagger$ & 1.00 & 0.84 & $0.60,1.18$ & 0.91 & $0.65,1.28$ & 0.75 & $0.53,1.05$ & 0.14 \\
\hline Multivariate model $2 \S$ & 1.00 & 0.86 & $0.61,1.29$ & 0.94 & $0.64,1.37$ & 0.80 & $0.55,1.17$ & 0.30 \\
\hline Multivariate model 3॥ & 1.00 & 0.89 & $0.61,1.31$ & 0.95 & $0.65,1.39$ & 0.77 & $0.52,1.12$ & 0.19 \\
\hline \multicolumn{9}{|l|}{ Isoflavones } \\
\hline Median† & 0.85 & \multicolumn{2}{|c|}{$2 \cdot 23$} & \multicolumn{2}{|c|}{6.02} & \multicolumn{2}{|c|}{$19 \cdot 6$} & \\
\hline IQR & $0.62-1.14$ & \multicolumn{2}{|c|}{$1.81-2.98$} & \multicolumn{2}{|c|}{$4 \cdot 67-8 \cdot 12$} & \multicolumn{2}{|c|}{$13 \cdot 9-31 \cdot 8$} & \\
\hline Cases/control & $162 / 141$ & \multicolumn{2}{|c|}{$132 / 141$} & \multicolumn{2}{|c|}{$130 / 141$} & \multicolumn{2}{|c|}{$140 / 141$} & \\
\hline Multivariate model $1 \ddagger$ & 1.00 & 0.81 & $0.58,1.13$ & 0.77 & $0.55,1.08$ & 0.84 & $0.60,1.17$ & 0.68 \\
\hline Multivariate model $2 \S$ & 1.00 & 0.76 & $0.52,1.11$ & 0.77 & $0.53,1.12$ & 0.82 & $0.56,1.19$ & 0.72 \\
\hline Multivariate model $3 \|$ & 1.00 & 0.76 & $0.52,1.11$ & 0.77 & $0.52,1.13$ & 0.78 & $0.54,1.14$ & 0.53 \\
\hline \multicolumn{9}{|l|}{ Lignans } \\
\hline Median† & 0.13 & \multicolumn{2}{|c|}{1.01} & \multicolumn{2}{|c|}{$2 \cdot 71$} & \multicolumn{2}{|c|}{$7 \cdot 23$} & \\
\hline IQR & $0.10-0.27$ & \multirow{2}{*}{\multicolumn{2}{|c|}{$\begin{array}{c}0.74-1.34 \\
124 / 141\end{array}$}} & \multirow{2}{*}{\multicolumn{2}{|c|}{$\begin{array}{c}2 \cdot 20-3 \cdot 42 \\
141 / 141\end{array}$}} & \multirow{2}{*}{\multicolumn{2}{|c|}{$\begin{array}{c}5 \cdot 37-10 \cdot 6 \\
140 / 141\end{array}$}} & \\
\hline Cases/control & $159 / 141$ & & & & & & 1141 & \\
\hline Multivariate model $1 \ddagger$ & 1.00 & 0.78 & $0.56,1.09$ & 0.92 & $0.68,1.25$ & 0.86 & $0.61,1.22$ & 0.75 \\
\hline Multivariate model $2 \S$ & 1.00 & 0.89 & $0.61,1.29$ & 1.09 & $0.77,1.54$ & 0.97 & $0.66,1.42$ & 0.92 \\
\hline Multivariate model $3 \|$ & 1.00 & 0.87 & $0.60,1.27$ & $1 \cdot 10$ & $0.78,1.57$ & 0.93 & $0.63,1.38$ & 0.95 \\
\hline
\end{tabular}

* Linear trend was tested by treating the median of quartiles as a continuous variable.

$\dagger$ the values are expressed as $\mathrm{nmol} / \mathrm{mg}$ creatinine.

‡ Multivariate model 1: adjusted for age at biospecimen collection (continuous) and fasting duration ( $\leq 6$ and $>6 \mathrm{~h}$ ).

$\S$ Multivariate model 2: further adjusted for education level, BMI, physical activity, smoking status, alcohol use, hypertension history and total energy intake.

II Multivariate model 3: further adjusted for vegetable intake, fruits and related juices, SFA and $n-3$ fatty acid.

was particularly evident in the subgroup of cases with HbA1c $\geq 6.5 \%$, but not in those with HbA1c $<6.5 \%$. When the second to fourth quartiles were combined, the OR comparing with Q1 was $0.77(95 \% \mathrm{CI} 0.56,1.06 ; P=0.11)$ in the total study sample, 0.89 (95\% CI $0.56,1.41 ; P=0.62)$ in the subgroup with HbA1c $<6.5 \%$ at baseline and 0.53 (95\% CI 0.32, $0 \cdot 88 ; P=0.01)$ in the subgroup of cases with HbA1c $\geq 6.5 \%$ (data not shown).

The associations between subclasses of urine metabolites of isoflavones and lignans and T2D risk are presented in online Supplementary Table S2. Although no statistically significant trend was observed, the second quartile of daidzein and third quartile of genistein were significantly associated with a lower risk of T2D compared with the corresponding first quartile.
No statistically significant trend was observed in the strata of cases with high or low HbA1c levels (data not shown).

\section{Discussion}

In this prospective investigation among middle-aged and elderly Chinese, we found no significant associations between urine phyto-oestrogen metabolites, either isoflavones or lignans, and risk of T2D. However, some intermediate concentrations of daidzein and genistein were associated with lower risk. In subjects with $\mathrm{HbA1c} \geq 6.5 \%$ at baseline, higher levels of urinary isoflavone metabolites were associated with a reduced risk, although no dose-response relationship was observed. 
Table 4. Incident type 2 diabetes according to total urine isoflavone and lignan metabolites: stratified by HbA1c level (Odds ratios and $95 \%$ confidence intervals; medians and interquartile ranges (IQR))

\begin{tabular}{|c|c|c|c|c|c|c|c|c|}
\hline & \multicolumn{7}{|c|}{ Quartiles (Q) } & \multirow[b]{3}{*}{$P_{\text {for trend }}$} \\
\hline & \multirow[b]{2}{*}{ Q1 } & \multicolumn{2}{|c|}{ Q2 } & \multicolumn{2}{|c|}{ Q3 } & \multicolumn{2}{|c|}{ Q4 } & \\
\hline & & OR & $95 \% \mathrm{Cl}$ & OR & $95 \% \mathrm{Cl}$ & OR & $95 \% \mathrm{Cl}$ & \\
\hline \multicolumn{9}{|l|}{ Isoflavones } \\
\hline \multicolumn{9}{|l|}{$\mathrm{HbA} 1 \mathrm{c}<6.5 \%$} \\
\hline Median† & 0.85 & \multirow{2}{*}{\multicolumn{2}{|c|}{$\begin{array}{c}2.33 \\
1.84-3.14\end{array}$}} & & & \multicolumn{2}{|c|}{$19 \cdot 2$} & \\
\hline IQR & $0.58-1.14$ & & & & $-8 \cdot 33$ & \multirow{2}{*}{\multicolumn{2}{|c|}{$\begin{array}{c}14.0-31 \cdot 8 \\
75 / 80\end{array}$}} & \\
\hline Cases/control & $73 / 70$ & \multicolumn{2}{|c|}{$68 / 71$} & \multicolumn{2}{|c|}{$70 / 65$} & & & \\
\hline Multivariate model $1 \ddagger$ & 1.00 & 0.90 & $0.57,1.44$ & 0.97 & $0.61,1.54$ & 0.89 & $0.57,1.39$ & 0.71 \\
\hline Multivariate model $2 \S$ & 1.00 & 0.82 & $0.47,1.41$ & 1.05 & $0.60,1.83$ & 0.88 & $0.52,1.49$ & 0.80 \\
\hline Multivariate model 3॥ & 1.00 & 0.80 & $0.46,1.39$ & 1.03 & $0.59,1.81$ & 0.83 & $0.48,1.42$ & 0.65 \\
\hline \multicolumn{9}{|l|}{$\mathrm{HbA} 1 \mathrm{c} \geq 6.5 \%$} \\
\hline Median† & 0.84 & \multicolumn{2}{|c|}{$2 \cdot 11$} & \multicolumn{2}{|c|}{6.07} & \multicolumn{2}{|c|}{$20 \cdot 1$} & \\
\hline IQR & $0.65-1.14$ & \multirow{2}{*}{\multicolumn{2}{|c|}{$\begin{array}{c}1.74-2 \cdot 72 \\
64 / 70\end{array}$}} & \multirow{2}{*}{\multicolumn{2}{|c|}{$\begin{array}{c}4 \cdot 66-7 \cdot 78 \\
60 / 76\end{array}$}} & \multirow{2}{*}{\multicolumn{2}{|c|}{$\begin{array}{c}13 \cdot 9-32 \cdot 6 \\
65 / 61\end{array}$}} & \\
\hline Cases/control & $89 / 71$ & & & & & & & \\
\hline Multivariate model $1 \ddagger$ & 1.00 & 0.71 & $0.43,1.14$ & 0.60 & $0.37,0.98$ & 0.83 & $0.50,1.37$ & 0.99 \\
\hline Multivariate model $2 \S$ & 1.00 & 0.63 & $0.36,1.10$ & 0.55 & $0.32,0.94$ & 0.71 & $0.40,1.26$ & 0.74 \\
\hline Multivariate model 3॥ & 1.00 & 0.60 & $0.34,1.07$ & 0.52 & $0.30,0.92$ & 0.64 & $0.35,1.16$ & 0.50 \\
\hline \multicolumn{9}{|l|}{ Lignans } \\
\hline \multicolumn{9}{|l|}{$\mathrm{HbA} 1 \mathrm{c}<6.5 \%$} \\
\hline Median† & 0.13 & \multirow{2}{*}{\multicolumn{2}{|c|}{$\begin{array}{c}1.02 \\
0.76-1.34\end{array}$}} & \multicolumn{2}{|c|}{$2 \cdot 73$} & \multicolumn{2}{|c|}{$7 \cdot 51$} & \\
\hline IQR & $0.11-0.26$ & & & & -3.40 & & $-10 \cdot 3$ & \\
\hline Cases/control & $84 / 70$ & \multicolumn{2}{|c|}{$63 / 75$} & \multicolumn{2}{|c|}{$65 / 71$} & & /70 & \\
\hline Multivariate model $1 \ddagger$ & 1.00 & 0.75 & $0.47,1.20$ & 0.86 & $0.55,1.35$ & 0.94 & $0.58,1.55$ & 0.83 \\
\hline Multivariate model $2 \S$ & 1.00 & 0.96 & $0.55,1.66$ & 1.08 & $0.63,1.85$ & $1 \cdot 18$ & $0.66,2.10$ & 0.49 \\
\hline Multivariate model 3॥ & 1.00 & 0.97 & $0.55,1.69$ & 1.08 & $0.63,1.85$ & $1 \cdot 18$ & $0.66,2 \cdot 12$ & 0.49 \\
\hline $\mathrm{HbA} 1 \mathrm{c} \geq 6.5 \%$ & & & & & & & & \\
\hline Median† & 0.15 & & & & & & 90 & \\
\hline IQR & $0.09-0.27$ & & -1.32 & & -3.46 & & $-12 \cdot 0$ & \\
\hline Cases/control & $75 / 71$ & & 66 & & & & /71 & \\
\hline Multivariate model $1 \ddagger$ & 1.00 & 0.85 & $0.53,1.38$ & 1.02 & $0.66,1.57$ & 0.87 & $0.53,1.43$ & 0.77 \\
\hline Multivariate model $2 \S$ & 1.00 & 0.89 & $0.53,1.51$ & 1.17 & $0.73,1.89$ & 0.89 & $0.51,1.53$ & 0.80 \\
\hline Multivariate model 3॥ & 1.00 & 0.86 & $0.49,1.48$ & $1 \cdot 19$ & $0.73,1.94$ & 0.87 & $0.50,1.52$ & 0.79 \\
\hline
\end{tabular}

* Linear trend was tested by treating the median of quartiles as a continuous variable.

† The values are expressed as $\mathrm{nmol} / \mathrm{mg}$ creatinine; $\mathrm{HbA} 1 \mathrm{c}$ of $6.5 \%$ equals $47.5 \mathrm{mmol} / \mathrm{mol}$.

$\ddagger$ Multivariate model 1: adjusted for age at biospecimen collection (continuous) and fasting duration ( $\leq 6$ and $>6 \mathrm{~h}$ ).

$\S$ Multivariate model 2: further adjusted for education level, BMI, physical activity, smoking status, alcohol use, hypertension history and total energy intake.

II Multivariate model 3: further adjusted for vegetable intake, fruits and related juices, SFA and $n-3$ fatty acid.

The association between dietary soya food intake (major sources of isoflavones) and T2D was assessed in a few longitudinal studies with contradictory findings ${ }^{(7,9)}$ or null associations $^{(8,11)}$ in different populations. In the previous report from SCHS, we also found an inverse association between unsweetened soya product intake and T2D risk ${ }^{(10)}$. These inconsistent findings from those studies may be due to the methodology challenges of accurately measuring dietary intakes ${ }^{(22)}$ or wide inter-individual variations in phyto-oestrogen metabolism ${ }^{(23)}$. In contrast, urine concentration of phyto-oestrogen metabolites has been preferred as an integrated measure of intake, absorption and metabolism.

In contrast to the null association in our study, a nested case-control study in a Korean population has recently reported an inverse association between serum genistein and T2D in women (316 cases) but not in men (377 cases) ${ }^{(24)}$; however, no significant association was found for dietary isoflavone intake ${ }^{(24)}$. Overnight urine was suggested to perform better than morning serum in estimating long-term soya intake $^{(25,26)}$. This is possibly because the dynamic changes of serum concentrations of isoflavones are minimised in urine assessments with longer half-life ${ }^{(27)}$ and stable content of isoflavones ${ }^{(28)}$. However, it is unclear whether use of serum rather than urine samples can explain the inconsistent findings between the two studies. In addition, HbA1c status of participants was not considered in the Korean study ${ }^{(24)}$.

A marginally significant inverse association was previously reported between dietary isoflavones and diabetes risk in SCHS, but it emerged only after adjustment for sweetened soya items ${ }^{(10)}$, indicating the importance of food context and preparation method. In our present analysis, the results did not change after further adjustment for sweetened soya items (data not shown). The null association between urine isoflavones and T2D risk also suggests that other components of soya, rather than the isoflavones per se, may account for the potential anti-diabetic effects. In a meta-analysis of clinical trials, fasting glucose reduction was observed in the subgroup of trials that used whole soya foods rather than isoflavone extracts or isolated soya protein ${ }^{(29)}$. However, another meta-analysis of clinical trials using high-dose isoflavone supplementation in non-Asian postmenopausal women found a moderate reduction in fasting glucose and insulin ${ }^{(30)}$. It is widely acknowledged 
that Asian populations consume much higher levels of soya foods as compared with non-Asian populations ${ }^{(3,5)}$. Therefore, it is possible that soya isoflavone supplements may benefit populations with traditionally very low soya intake, but not in populations where soya foods are commonly consumed a hypothesis that requires further testing. Moreover, it is also unclear whether the short-term glucose-lowering effect in those clinical trials ${ }^{(30)}$ could be extended to the long-term reduction of diabetes incidence in populations.

A nested case-control study within the Nurse's Health Study (NHS) has recently reported that urine concentrations of lignan metabolites were inversely associated with incident T2D ${ }^{(13)}$. We found no significant dose-dependent association in this Asian population. However, in the NHS study, the inverse association was only evident in the younger NHS II cohort with a mean age of 45.4 years, but not in the older NHS cohort with a mean age of 65.5 years ${ }^{(13)}$. In our study, the mean age of the participants at the time of urine sample collection was $59 \cdot 8$ years. Therefore, it is possible that lignans may have an effect on early-onset but not late-onset of diabetes. Furthermore, no statistically significant association was observed between dietary lignan intake and T2D risk in European populations ${ }^{(11)}$. The effects of lignan supplementation on glucose homoeostasis have been examined in a number of clinical trials, and the results have also been inconsistent ${ }^{31,32)}$.

It has been widely acknowledged that various types of tofu and soyabean milk are the major sources of dietary isoflavone in Chinese populations ${ }^{(33)}$. In a previous report from the SCHS ${ }^{(16)}$, soya intake frequency was associated with urine isoflavone in a dose-dependent manner; this has been confirmed in our current analysis as well. On the other hand, lignans are ubiquitously present in many plant-based foods with flaxseed having the highest concentration (but flaxseed is not commonly consumed). Studies in European countries have found that cereals and grain products, vegetables, fruit and berries, and beverages are the major source of total lignan intake ${ }^{(34,35)}$. Limited studies in Chinese populations have suggested that tea consumption might be a potential dietary source $^{(36,37)}$, but the major food sources remain unclear. The urine concentration of isoflavones in our study (geometric mean $4.01 \mathrm{nmol} / \mathrm{mg}$ creatinine) was slightly lower compared with a study in Shanghai men and women $(14.8-18.2 \mathrm{nmol} / \mathrm{mg}$ creatinine $)^{(38)}$. This might be because dietary soya consumption levels were generally higher in Shanghai (average $30.5 \mathrm{mg} / \mathrm{d}$ soya isoflavone intake $)^{(37)}$ compared with our population (average $15.8 \mathrm{mg} / \mathrm{d})^{(39)}$, and they had measured a few more low-abundance isoflavone metabolites. Urine total lignan concentrations in our study $(1.71 \mathrm{nmol} / \mathrm{mg}$ creatinine) were comparable with the NHS cohort $(2.32 \mathrm{nmol} / \mathrm{mg} \text { creatinine })^{(13)}$, although slightly higher than the Shanghai women (about $0.60 \mathrm{nmol} / \mathrm{mg}$ creatinine) ${ }^{(40)}$.

There are several potential limitations to our study that we should acknowledge. First, we only collected morning spot urine samples, and this might not be representative of phytooestrogen concentrations in individuals compared with 24-h samples. Collecting 24-h urine samples was not feasible in our study, as in other large epidemiological studies ${ }^{(12,13,38)}$. However, a high correlation was seen between overnight spot urine and 24-h urine samples for enterolactone recovery ${ }^{(41)}$, and spot urine had high agreement with overnight urine for isoflavone levels ${ }^{(40)}$. Several studies have also confirmed a reasonably good correlation between dietary soya intakes and urine concentrations of isoflavone metabolites ${ }^{(16,42,43)}$. Second, we only obtained a single measurement of urine isoflavones and lignans, and this may not reflect long-term exposure status. The plasma elimination half-lives of isoflavones ${ }^{(44)}$ and lignans ${ }^{(45)}$ are almost half a day or less. The within-person stability of urine lignan metabolites were in the acceptable range in Caucasians over $1-2$ years ${ }^{(13)}$, but such data for isoflavones are scarce, particularly in Chinese populations with high intake levels. Future studies may consider multiple assessments over time to get more accurate information about phyto-oestrogen exposure. Altogether, the measurement errors of isoflavones and lignans are more likely to be non-differential in our prospective investigation, and thus may attenuate our results towards null. Third, at the time of urine collection, a group of cases already had high HbA1c levels that met the current diabetes diagnosis criteria, even though they did not report a diagnosis of T2D. Although we have stratified the analysis by baseline HbA1c levels, the sample size in each stratum was substantially reduced and that analysis might have been underpowered. Finally, selection bias by loss to follow-up in the cohort is possible but may have minimal impact on our findings because the differences between groups with and without loss to follow-up were generally small (data not shown).

In conclusion, our prospective nested case-control study in a Chinese population provides little evidence of an association between urine isoflavones and lignans measured using a single collected specimen and risk of T2D in either women or men. Further studies are needed to confirm our results in other populations and to investigate the effects of different food sources of isoflavones and lignans on risk of T2D.

\section{Acknowledgements}

The authors thank Siew-Hong Low of the National University of Singapore for supervising the field work of the SCHS. Finally, the authors acknowledge the founding, long-standing principal investigator of the SCHS, Mimi C. Yu.

The SCHS was supported by the National Institutes of Health, USA (NCI R01 CA144034 and UM1 CA182876). This project was supported by Singapore MOE Tier 1 grant and MOH NMRC grant (CIRG/1354/2013).

A. P., W. P. K. and C. N. O.: designed and conducted the research; M. T. and B. L. L.: performed laboratory measurements; M. T.: analysed data and wrote the first draft; A. P.: critically revised the manuscript; W. P. K., R. M. v. D. and J. M. Y.: assisted in interpreting the data and edited the manuscript; A. P. had primary responsibility for the final content. All authors read and approved the final version of the manuscript.

The authors declare that there are no conflicts of interest.

\section{Supplementary material}

For supplementary material/s referred to in this article, please visit http://dx.doi.org/doi:10.1017/S0007114516000581 


\section{References}

1. Kwon DY, Daily JW 3rd, Kim HJ, et al. (2010) Antidiabetic effects of fermented soybean products on type 2 diabetes. Nutr Res 30, 1-13.

2. Talaei M \& Pan A (2015) Role of phytoestrogens in prevention and management of type 2 diabetes. World $J$ Diabetes 6 271-283.

3. Cederroth CR \& Nef S (2009) Soy, phytoestrogens and metabolism: a review. Mol Cell Endocrinol 304, 30-42.

4. Adolphe JL, Whiting SJ, Juurlink BH, et al. (2010) Health effects with consumption of the flax lignan secoisolariciresinol diglucoside. Br J Nutr 103, 929-938.

5. Behloul N \& Wu G (2013) Genistein: a promising therapeutic agent for obesity and diabetes treatment. Eur J Pharmacol 698, $31-38$.

6. Gilbert ER \& Liu D (2013) Anti-diabetic functions of soy isoflavone genistein: mechanisms underlying its effects on pancreatic beta-cell function. Food Funct 4, 200-212.

7. Villegas R, Gao YT, Yang G, et al. (2008) Legume and soy food intake and the incidence of type 2 diabetes in the Shanghai Women's Health Study. Am J Clin Nutr 87, $162-167$.

8. Nanri A, Mizoue T, Takahashi Y, et al. (2010) Soy product and isoflavone intakes are associated with a lower risk of type 2 diabetes in overweight Japanese women. J Nutr 140, 580-586.

9. Morimoto Y, Steinbrecher A, Kolonel LN, et al. (2011) Soy consumption is not protective against diabetes in Hawaii: the Multiethnic Cohort. Eur J Clin Nutr 65, 279-282.

10. Mueller NT, Odegaard AO, Gross MD, et al. (2012) Soy intake and risk of type 2 diabetes in Chinese Singaporeans [corrected]. Eur J Nutr 51, 1033-1040.

11. Zamora-Ros R, Forouhi NG, Sharp SJ, et al. (2013) The association between dietary flavonoid and lignan intakes and incident type 2 diabetes in European populations: the EPIC-InterAct study. Diabetes Care 36, 3961-3970.

12. Bredsdorff L, Obel T, Dethlefsen C, et al. (2013) Urinary flavonoid excretion and risk of acute coronary syndrome in a nested case-control study. Am J Clin Nutr 98, 209-216.

13. Sun Q, Wedick NM, Pan A, et al. (2014) Gut microbiota metabolites of dietary lignans and risk of type 2 diabetes: a prospective investigation in two cohorts of U.S. women. Diabetes Care 37, 1287-1295.

14. Hankin JH, Stram DO, Arakawa K, et al. (2001) Singapore Chinese Health Study: development, validation, and calibration of the quantitative food frequency questionnaire. Nutr Cancer 39, 187-195.

15. Koh WP, Yuan JM, Sun CL, et al. (2003) Angiotensin I-converting enzyme (ACE) gene polymorphism and breast cancer risk among Chinese women in Singapore. Cancer Res 63 , 573-578.

16. Seow A, Shi CY, Franke AA, et al. (1998) Isoflavonoid levels in spot urine are associated with frequency of dietary soy intake in a population-based sample of middle-aged and older Chinese in Singapore. Cancer Epidemiol Biomarkers Prev 7, $135-140$.

17. Odegaard AO, Koh WP, Arakawa K, et al. (2010) Soft drink and juice consumption and risk of physician-diagnosed incident type 2 diabetes: the Singapore Chinese Health Study. Am J Epidemiol 171, 701-708.

18. Chen Z, Pereira MA, Seielstad M, et al. (2014) Joint effects of known type 2 diabetes susceptibility loci in genome-wide association study of Singapore Chinese: the Singapore Chinese Health Study. PLOS ONE 9, e87762.

19. Parker DL, Rybak ME \& Pfeiffer CM (2012) Phytoestrogen biomonitoring: an extractionless LC-MS/MS method for measuring urinary isoflavones and lignans by use of atmospheric pressure photoionization (APPI). Anal Bioanal Chem $\mathbf{4 0 2}$, $1123-1136$

20. International Expert Committee (2009) International Expert Committee report on the role of the $\mathrm{A} 1 \mathrm{C}$ assay in the diagnosis of diabetes. Diabetes Care 32, 1327-1334.

21. American Diabetes Association (2010) Diagnosis and classification of diabetes mellitus. Diabetes Care 33, Suppl. 1, S62-S69.

22. Cermak R, Durazzo A, Maiani G, et al. (2009) The influence of postharvest processing and storage of foodstuffs on the bioavailability of flavonoids and phenolic acids. Mol Nutr Food Res 53, Suppl. 2, S184-S193.

23. Franke AA, Halm BM, Kakazu K, et al. (2009) Phytoestrogenic isoflavonoids in epidemiologic and clinical research. Drug Test Anal 1, 14-21.

24. Ko KP, Kim CS, Ahn Y, et al. (2015) Plasma isoflavone concentration is associated with decreased risk of type 2 diabetes in Korean women but not men: results from the Korean Genome and Epidemiology Study. Diabetologia 58, 726-735.

25. Yamamoto S, Sobue T, Sasaki S, et al. (2001) Validity and reproducibility of a self-administered food-frequency questionnaire to assess isoflavone intake in a Japanese population in comparison with dietary records and blood and urine isoflavones. J Nutr 131, 2741-2747.

26. Fraser GE, Franke AA, Jaceldo-Siegl K, et al. (2010) Reliability of serum and urinary isoflavone estimates. Biomarkers $\mathbf{1 5}$, $135-139$.

27. Setchell KD \& Cole SJ (2006) Method of defining equolproducer status and its frequency among vegetarians. $J$ Nutr 136, 2188-2193.

28. Lee SA, Wen W, Xiang YB, et al. (2007) Assessment of dietary isoflavone intake among middle-aged Chinese men. J Nutr 137, 1011-1016.

29. Liu ZM, Chen YM \& Ho SC (2011) Effects of soy intake on glycemic control: a meta-analysis of randomized controlled trials. Am J Clin Nutr 93, 1092-1101.

30. Zhang YB, Chen WH, Guo JJ, et al. (2013) Soy isoflavone supplementation could reduce body weight and improve glucose metabolism in non-Asian postmenopausal women a meta-analysis. Nutrition 29, 8-14.

31. Zhang W, Wang X, Liu Y, et al. (2008) Dietary flaxseed lignan extract lowers plasma cholesterol and glucose concentrations in hypercholesterolaemic subjects. Br J Nutr 99, 1301-1309.

32. Billinsky J, Glew RA, Cornish SM, et al. (2013) No evidence of hypoglycemia or hypotension in older adults during 6 months of flax lignan supplementation in a randomized controlled trial: a safety evaluation. Pharm Biol 51, 778-782.

33. Chan SG, Ho SC, Kreiger N, et al. (2007) Dietary sources and determinants of soy isoflavone intake among midlife Chinese Women in Hong Kong. J Nutr 137, 2451-2455.

34. Zamora-Ros R, Knaze V, Lujan-Barroso L, et al. (2012) Dietary intakes and food sources of phytoestrogens in the European Prospective Investigation into Cancer and Nutrition (EPIC) 24-hour dietary recall cohort. Eur J Clin Nutr 66 , 932-941.

35. Tetens I, Turrini A, Tapanainen H, et al. (2013) Dietary intake and main sources of plant lignans in five European countries. Food Nutr Res 57, 10.3402/fnr.v57i0.19805.

36. Huang MH, Norris J, Han W, et al. (2012) Development of an updated phytoestrogen database for use with the SWAN food frequency questionnaire: intakes and food sources in a community-based, multiethnic cohort study. Nutr Cancer $\mathbf{6 4}$, 228-244.

37. Wu X, Cai H, Gao YT, et al. (2012) Correlations of urinary phytoestrogen excretion with lifestyle factors and dietary intakes among middle-aged and elderly Chinese women. Int J Mol Epidemiol Genet 3, 18-29. 
38. Zhang X, Gao YT, Yang G, et al. (2012) Urinary isoflavonoids and risk of coronary heart disease. Int $J$ Epidemiol $\mathbf{4 1}$, $1367-1375$.

39. Talaei M, Koh WP, van Dam RM, et al. (2014) Dietary soy intake is not associated with risk of cardiovascular disease mortality in Singapore Chinese adults. J Nutr 144, 921-928.

40. Franke AA, Hebshi SM, Pagano I, et al. (2010) Urine accurately reflects circulating isoflavonoids and ascertains compliance during soy intervention. Cancer Epidemiol Biomarkers Prev 19, $1775-1783$.

41. Krogholm KS, Bysted A, Brantsaeter AL, et al. (2012) Evaluation of flavonoids and enterolactone in overnight urine as intake biomarkers of fruits, vegetables and beverages in the Inter99 cohort study using the method of triads. Br J Nutr $\mathbf{1 0 8}$, 1904-1912.
42. Jaceldo-Siegl K, Fraser GE, Chan J, et al. (2008) Validation of soy protein estimates from a food-frequency questionnaire with repeated 24-h recalls and isoflavonoid excretion in overnight urine in a Western population with a wide range of soy intakes. Am J Clin Nutr 87, 1422-1427.

43. Tseng M, Olufade T, Kurzer MS, et al. (2008) Food frequency questionnaires and overnight urines are valid indicators of daidzein and genistein intake in U.S. women relative to multiple 24-h urine samples. Nutr Cancer 60, 619-626.

44. Manach C, Williamson G, Morand C, et al. (2005) Bioavailability and bioefficacy of polyphenols in humans. I. Review of 97 bioavailability studies. Am J Clin Nutr 81, 230S-242S.

45. Kuijsten A, Arts IC, Vree TB, et al. (2005) Pharmacokinetics of enterolignans in healthy men and women consuming a single dose of secoisolariciresinol diglucoside. J Nutr 135, 795-801. 